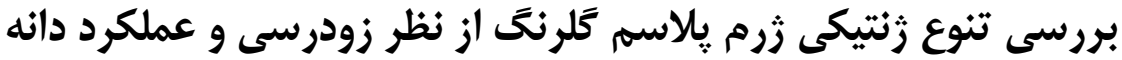

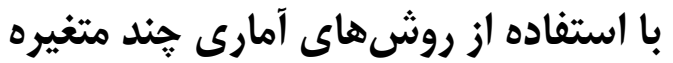

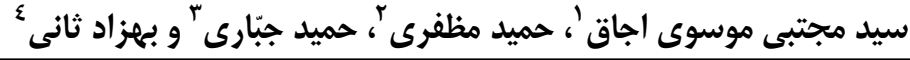

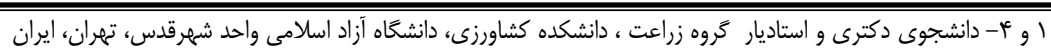

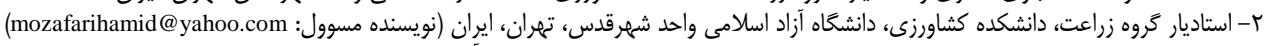

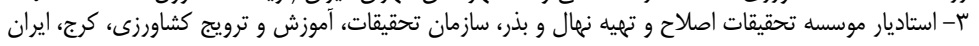 \\ تاريخ دريافت: \\ صف
}

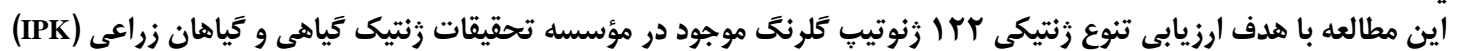

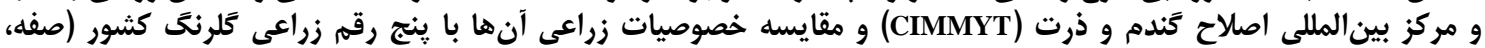

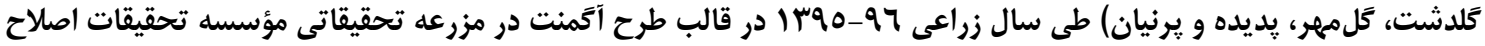

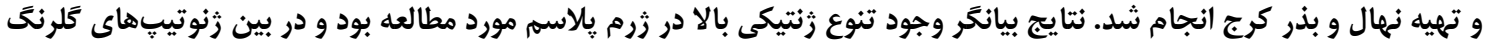

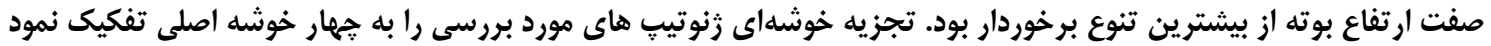

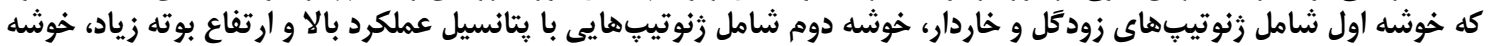

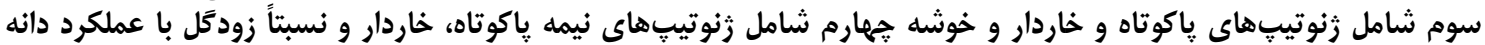

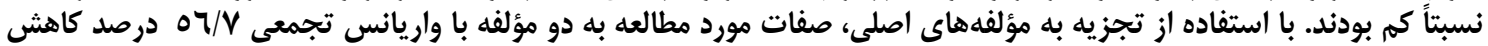

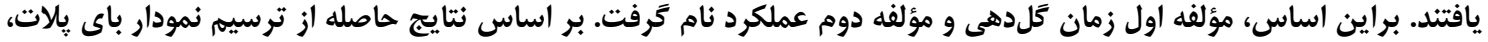

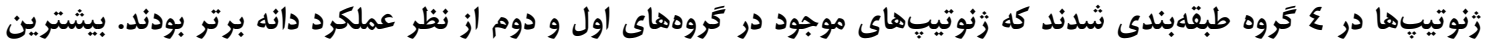

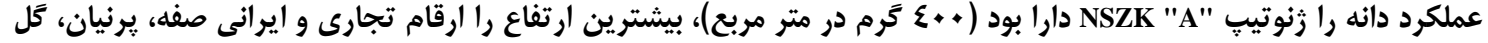

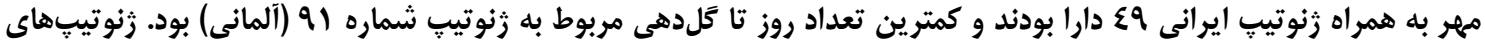

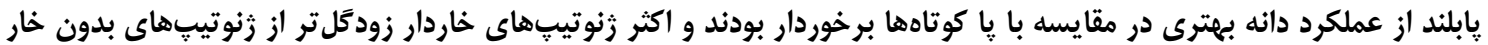

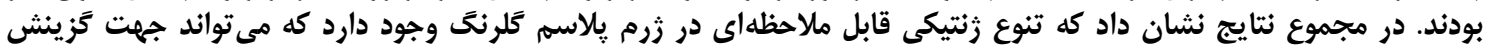

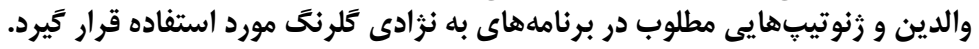

وازههاى كليدى: ارتفاع بوته، تجزيه باى يلات، تجزيه كلاستر، خار، زودكل

واريتهاى تجارى را براى اصلاحكر ميسر مىسازد (سا)..

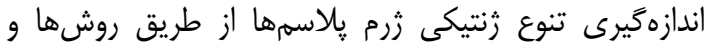

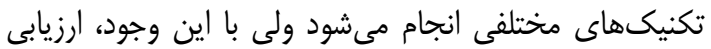

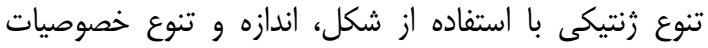

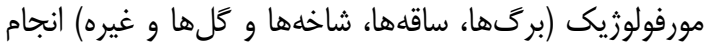

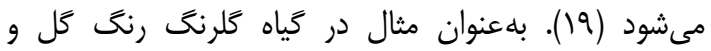

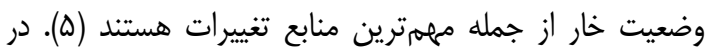

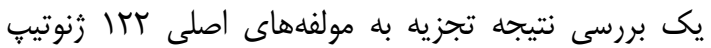

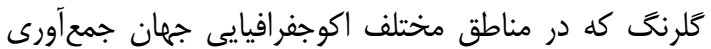

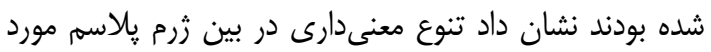

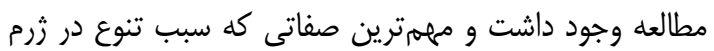

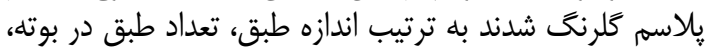

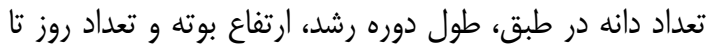
كلدهي بودند (19).

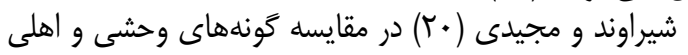

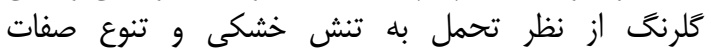

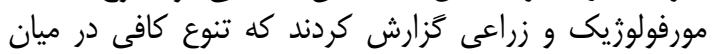

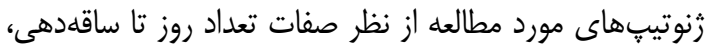

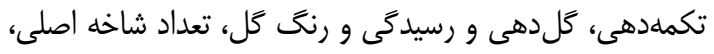

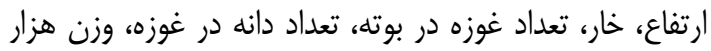
دانه، عملكرد دانه و درصد روغن دانه ورئه وجود دارد.

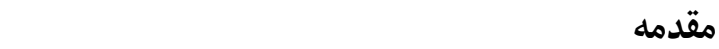

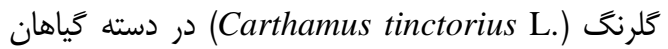

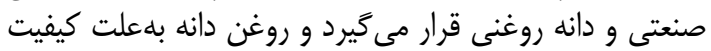

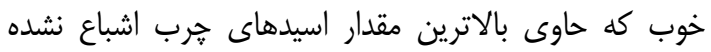

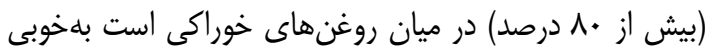

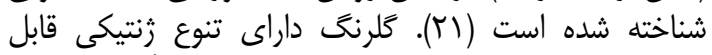

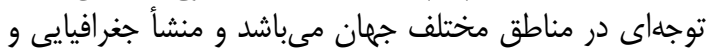

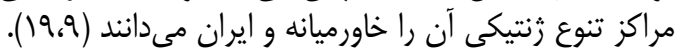

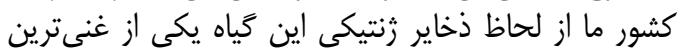

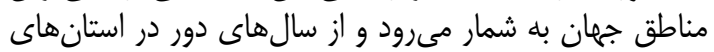

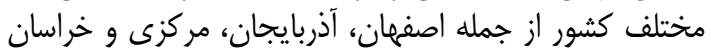

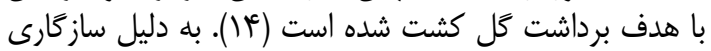

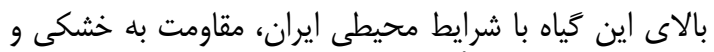

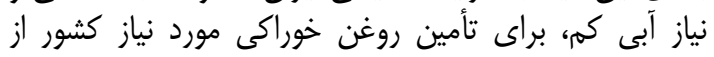

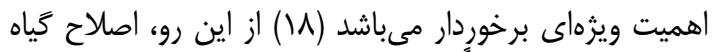

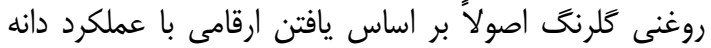

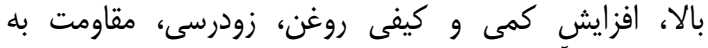

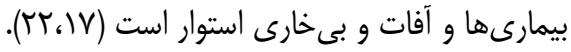

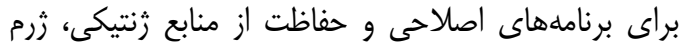

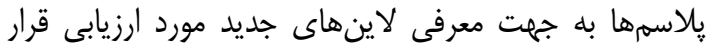

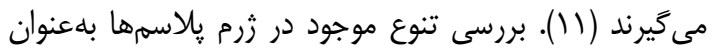

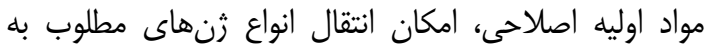


(19،1). بر اين اساس مطالعه تنوع زنتيكى و مقايسه

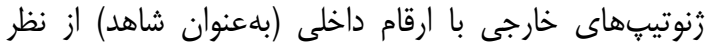

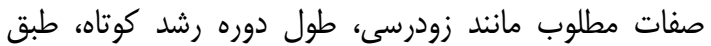

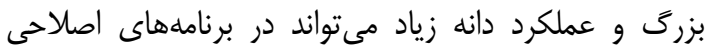

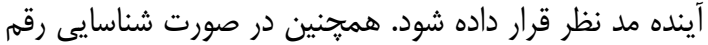

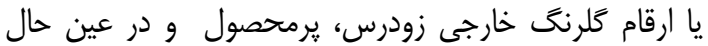

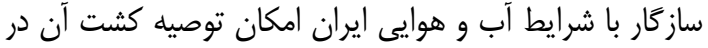

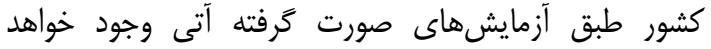

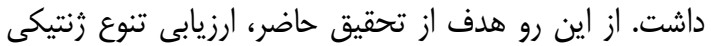

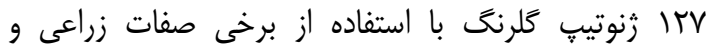

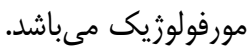

\section{مواد و روشها}

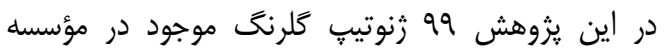

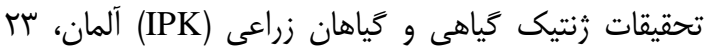

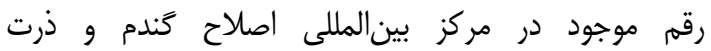

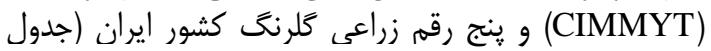

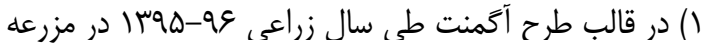

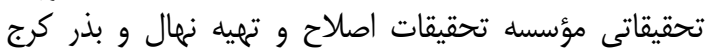

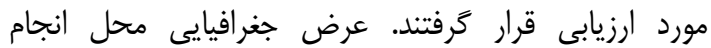

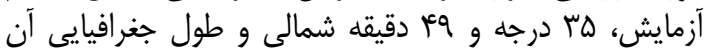

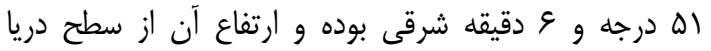

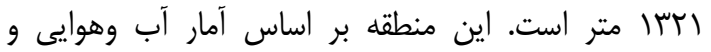

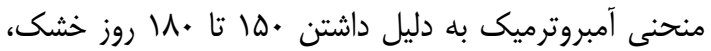

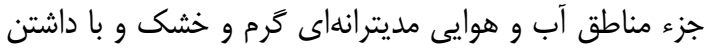

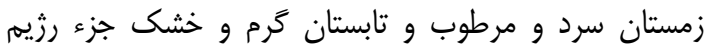

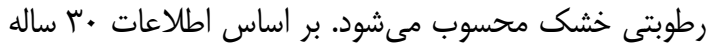

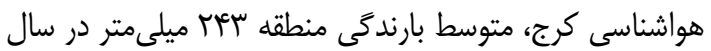

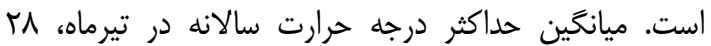

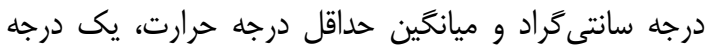

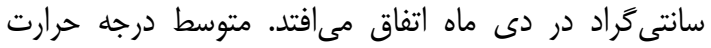

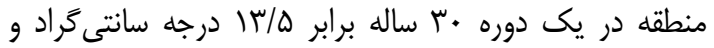

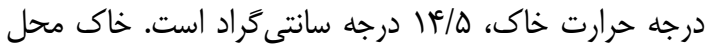

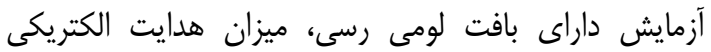

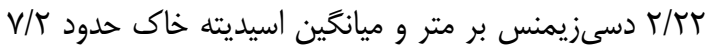

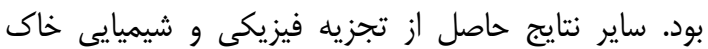
محل آزمايش در جدول اير ارائه شده است.

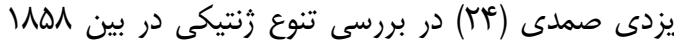

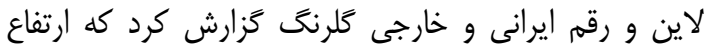

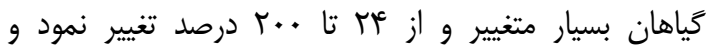

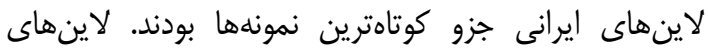

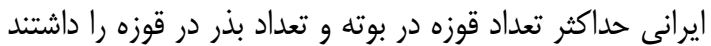

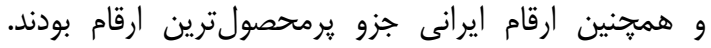

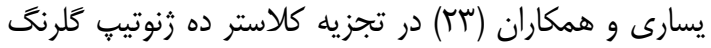

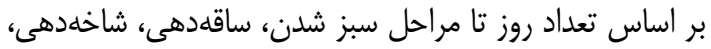

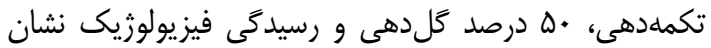

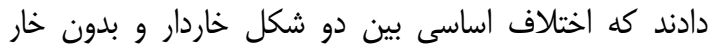

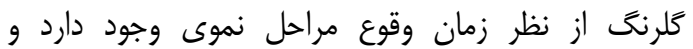

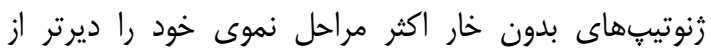

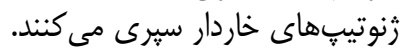

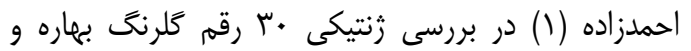

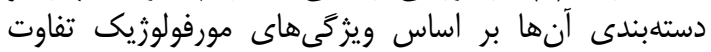

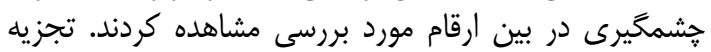

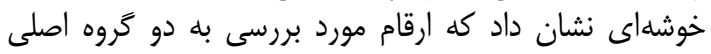

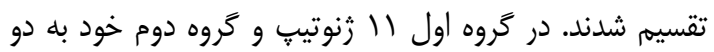

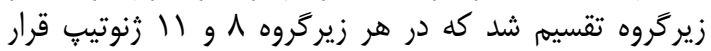

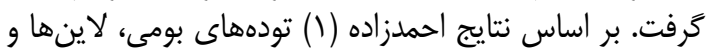

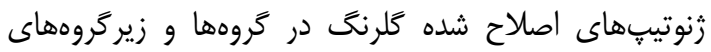

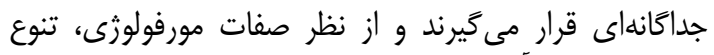
بسيارى در بين آنها وجود دارئران

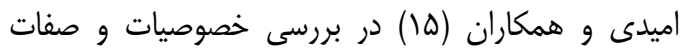

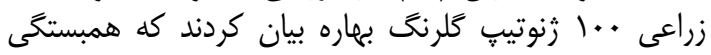

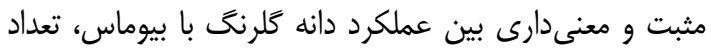

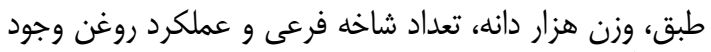

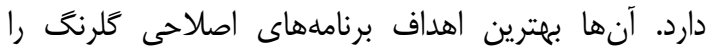

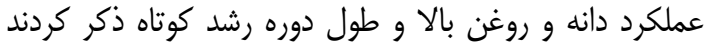

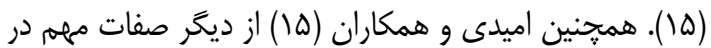

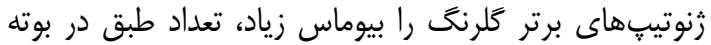

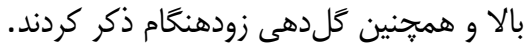

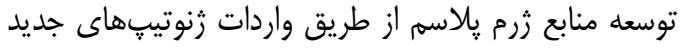

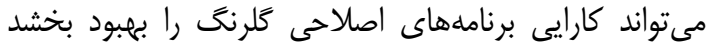

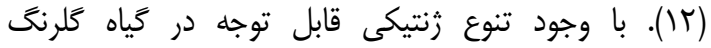

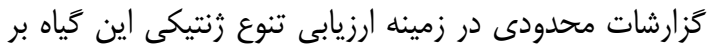

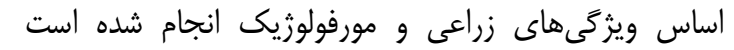

جدول ا- خصوصيات فيزيكوشيميايى خاك مزرعه

Table 1. Physicochemical properties of farm soil

\begin{tabular}{|c|c|c|c|c|c|c|c|c|c|c|}
\hline $\begin{array}{l}\text { سيلت } \\
\text { (\%) }\end{array}$ & إ) & ش) & 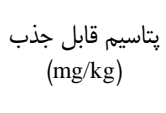 & فسفر قابل جذب & نيتروثن كل & (دسىزيمنس بر الكتريكى & كرآلى (درصد) & اسيديته خاك & بافت خاك & خاك \\
\hline 19 & Tr & re & TAS & $1 \pi / 9$ & .1 .9 & T/TT &.$/ Q \Lambda$ & $V / T r$ & لومى - رسى & . \\
\hline
\end{tabular}

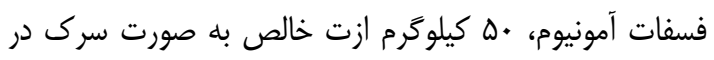

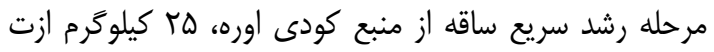

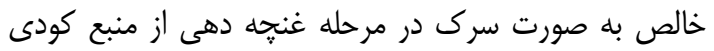

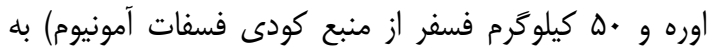
مزرعه اضافه شد.
بذر هر زنوتيّ در دو رديف به صورت جوى و رشته و به

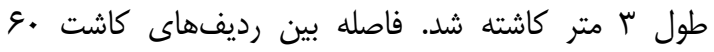

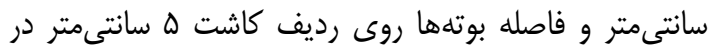

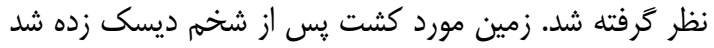

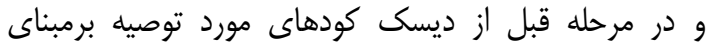

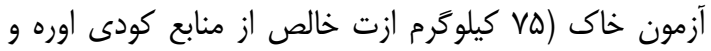


خوشهاى (كلاستر) به روش وارد' و بر اساس فاصله اقليدسى تجاي

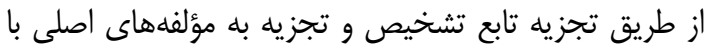

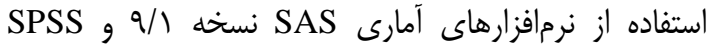

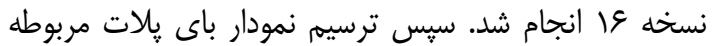
با استفاده از نرمافزار

\section{نتايج و بحث}

نتايج ضرايب همبستخى بين صفات صفات مورد مطالعه نشان

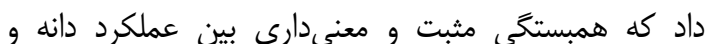

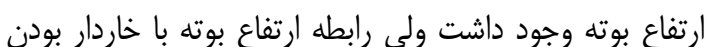

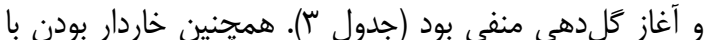

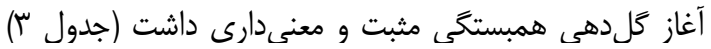

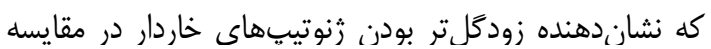

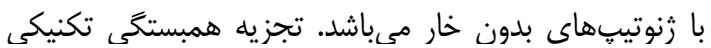

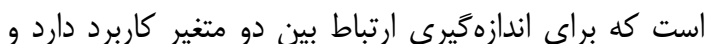

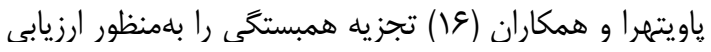

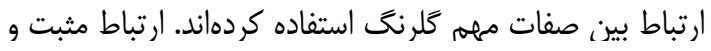

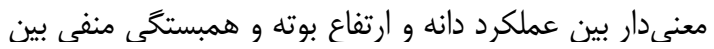

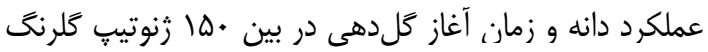

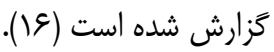

كشت در سب مهرماه انجام شد و با توجه به شرايط آب و هوايى يس از كاشت دو مرتبه آبيارى صورت كرات

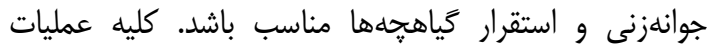

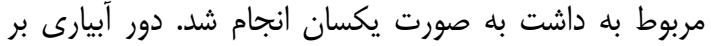

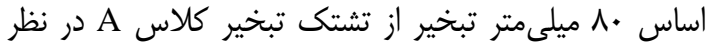

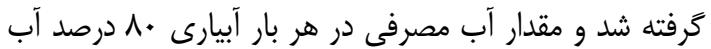

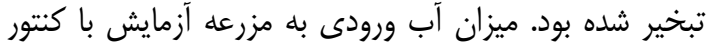

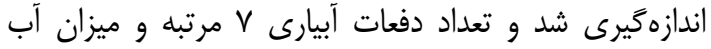

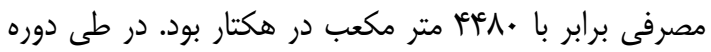

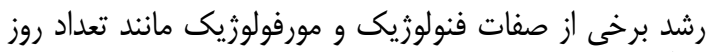

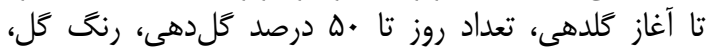

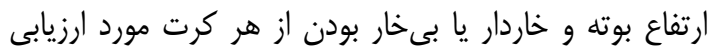

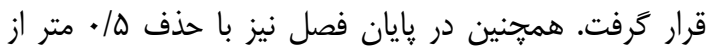

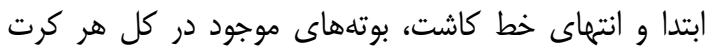

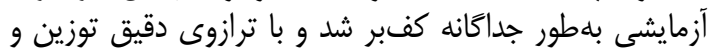

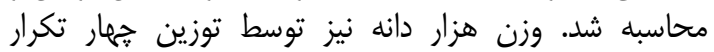

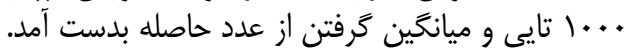

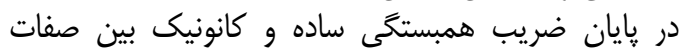

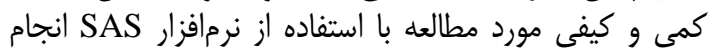
شد. همجنين براى تشخيص مود ميزان تمايز بين زنوتينها

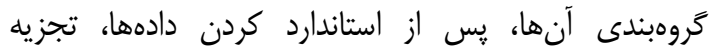

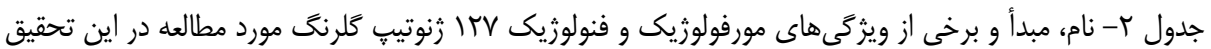
Table 2. Name, origin and some of morphologic and phonologic characteristics of 127 studied-safflower genotypes

\begin{tabular}{|c|c|c|c|c|c|c|c|c|}
\hline 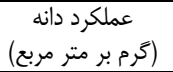 & ارتفاع بوته & وضعيت خار & كَل تعداد روز تامل & تعداد روز تا آغاز كلدهى & رنى گل & مبداً & نام زنوتيب & شماره زنوتيب \\
\hline $1 \cdot 1 / 0$ & متوسط & خاردار & ונוr & TRE & زرد & نامشخص & 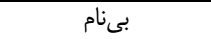 & 1 \\
\hline$V E / V$ & خيلى پاكوتاه & خاردار & rTV & 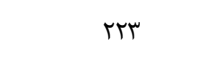 & زبه نارنجى مايل & ايتاليا & 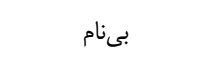 & r \\
\hline$v / \wedge$ & نيمه پاكوتاه & بدون خار & שTH & a & زرد & كانادا & Saffire & r \\
\hline ( & متوسط & بدون خار & ז & rqם & زرد & كانادا & AC Stirling & f \\
\hline$\Lambda F / \varepsilon$ & متوسط & بدون خار & THF & rqq & زرد & نامشخص & N006 & $\checkmark$ \\
\hline met/A & متوسط & بدون خار & ודץ & בצt & زرد & نامشخص & LEED & $\wedge$ \\
\hline TMT/S & متوسط & بدون خار & q & THE & زرد & نامشخص & N942 & 9 \\
\hline$r \cdots / \Lambda$ & متوسط & بدون خار & Fre & rq & زرد & نامشخص & NSZK "A" & 1. \\
\hline 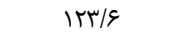 & ياكوتاه & بدون خار & • (זr. & THA & زرد & هند & KUSUM & 11 \\
\hline 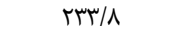 & ياكوتاه & بدون خار & מזr & rin & نارنجى & ايران & KOUSHEH & 19 \\
\hline$|0| / \bar{G}$ & ياكوتاه & خاردار & TTA & rt. & نارنجى & ياكستان & KUSAMBA & IV \\
\hline$\Lambda \mu / \cdot$ & متوسط & خاردار & ווזr & TrE & زرد & شوسياليستى & Färberdistel, Saflor & 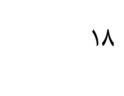 \\
\hline $\mathrm{Vq} / \mathrm{s}$ & ياكوتاه & خاردار & שr. & tTA & زرد & بلزيى & Färberdistel, Saflor & 19 \\
\hline$r \cdot / r$ & متوسط & خاردار & ווזץ & ב & زرد & آلمان & Färberdistel, Saflor & $r \cdot$ \\
\hline $1 \cdot V / 1$ & خيلى پاكوتاه & خاردار & THV & מצr & زرد & نامشخص & Färberdistel, Saflor & rI \\
\hline $11 V / 9$ & خيلى پاكوتاه & خاردار & trR & 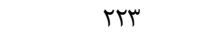 & نارنجى & آلمان & Färberdistel, Saflor & tr \\
\hline $91 / 8$ & ياكوتاه & خاردار & ווץ & TE & زرد - ت ا & مراكش & Färberdistel, Saflor & سץ \\
\hline
\end{tabular}

1- Ward 


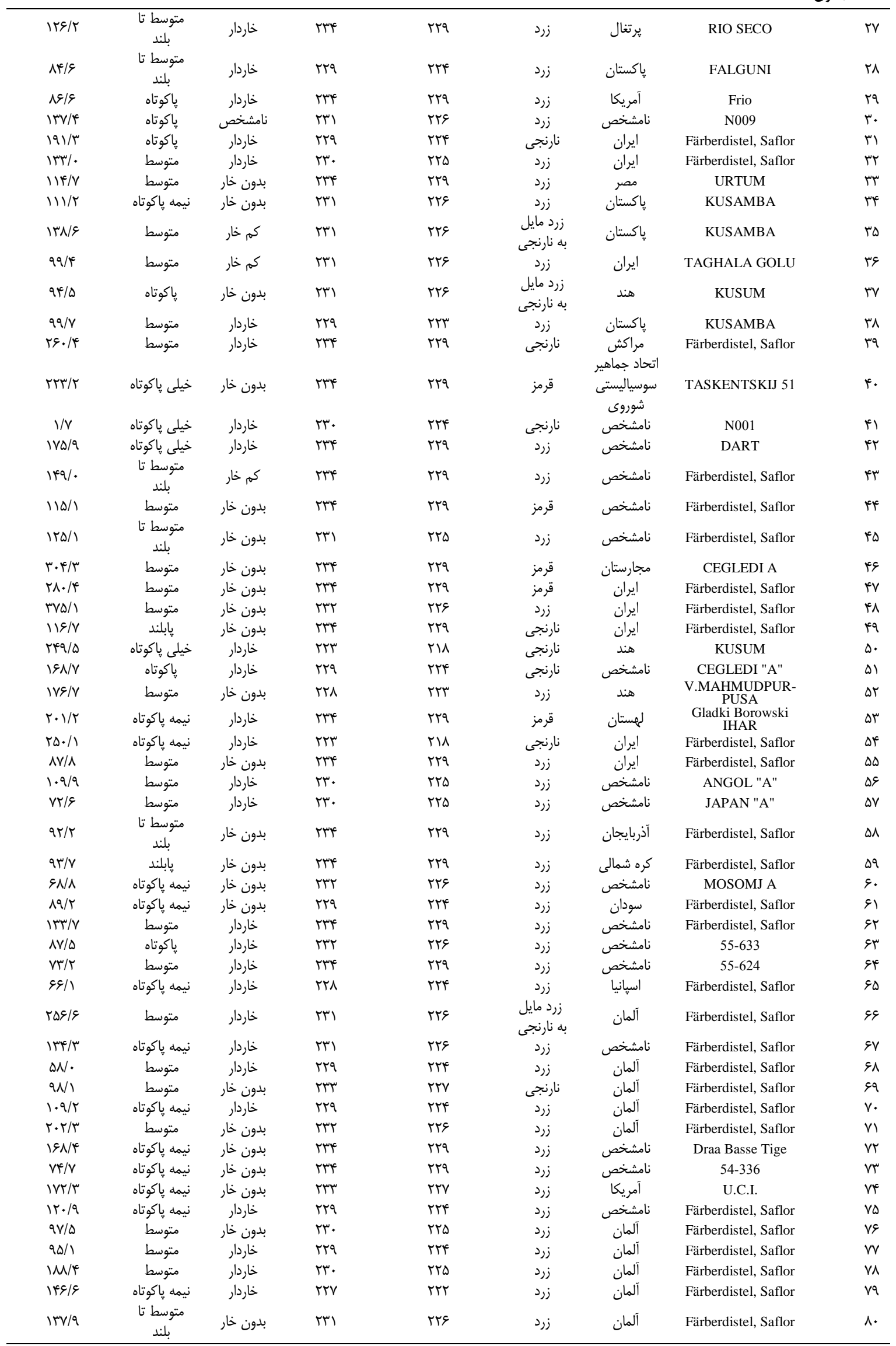




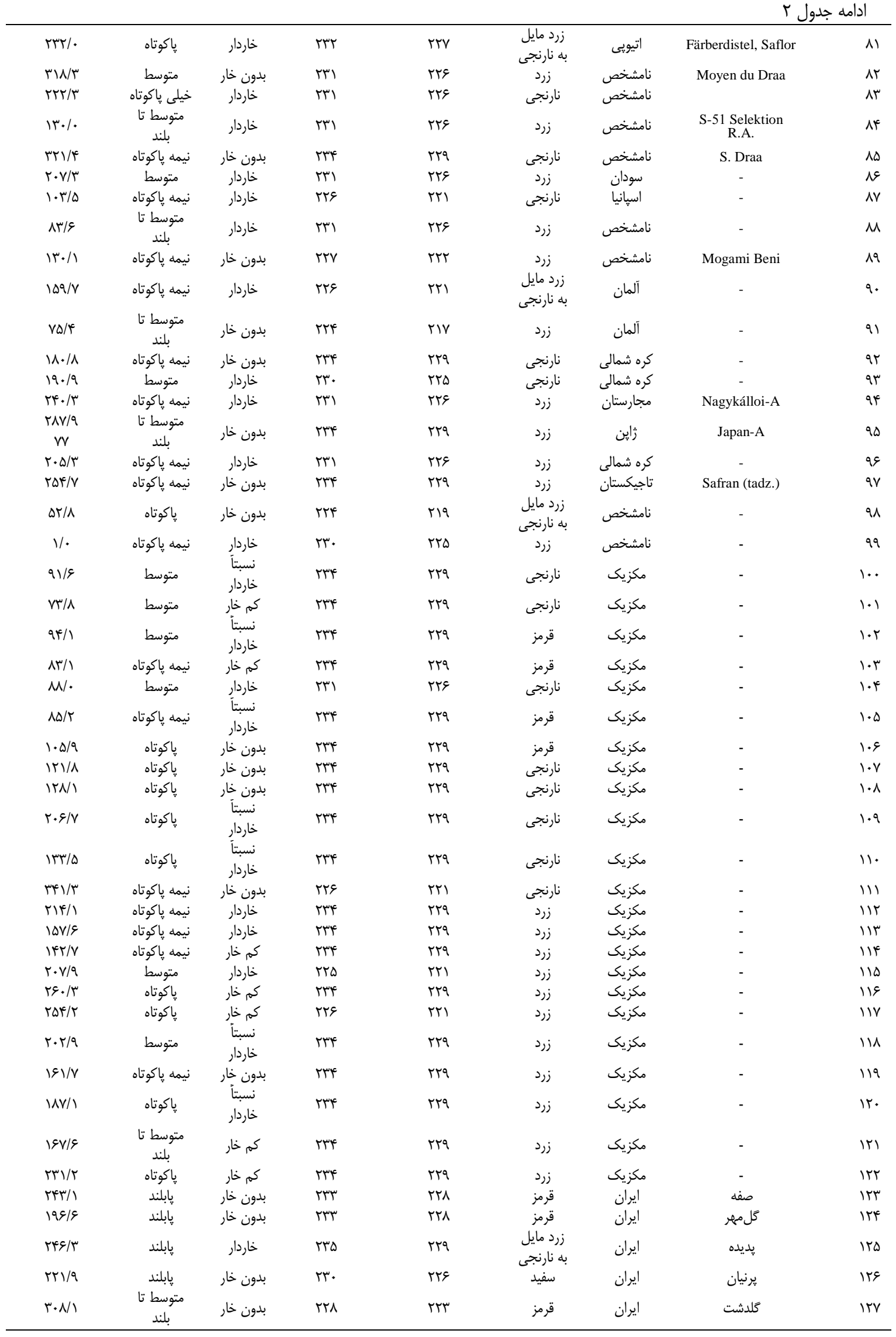




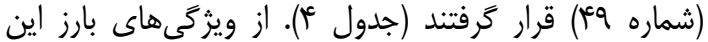

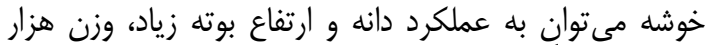

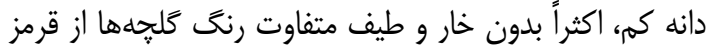

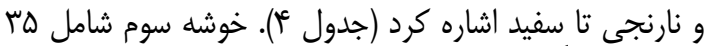

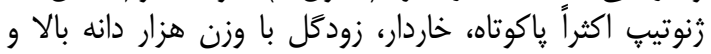

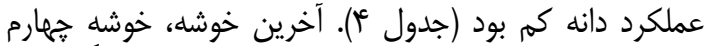

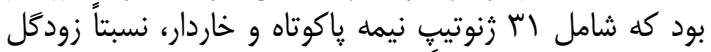

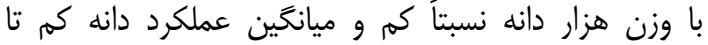

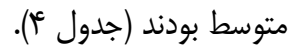

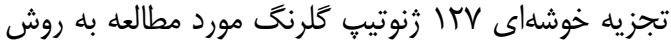

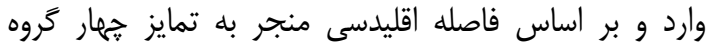

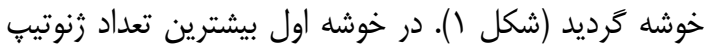

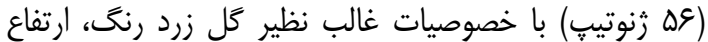

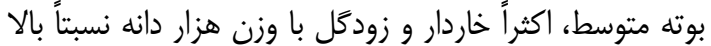

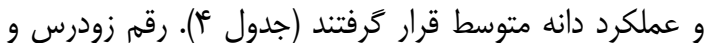

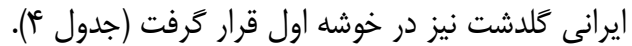

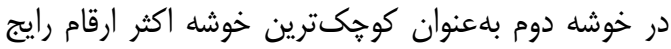

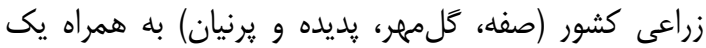

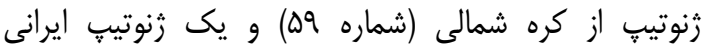

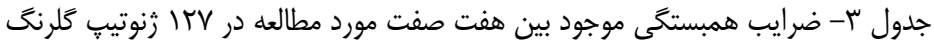
Table 3. The correlation coefficients between seven traits in 127 safflower genotypes

\begin{tabular}{|c|c|c|c|c|c|c|c|}
\hline عملكرد دانه & وزن هزار دانه & ارتفاع بوته & خاردار بودن & كلدهى كامل & آغاز كل دهى & رنگ كَل & صفات \\
\hline & & & & & & 1 & رنغ گل \\
\hline & & & & & 1 & $\cdot / / Q^{\mathrm{ns}}$ & آغاز كُل دهى \\
\hline & & & & 1 &.$/ 99^{* *}$ & $-\cdot / 1 \varepsilon^{\mathrm{ns}}$ & كل دهى كامل \\
\hline & & & 1 & . & $\cdot / V \Delta^{* *}$ & $\cdot /\left.r\right|^{\mathrm{ns}}$ & خاردار بودن \\
\hline & & 1 & $-.109^{* *}$ &.$- / 1 \& q^{*}$ & $-\cdot 10 \cdot^{*}$ & $\cdot / r^{\mathrm{ns}}$ & ارتفاع بوته \\
\hline & 1 & $-.19 V^{* *}$ & $\cdot 199^{* *}$ & $-\cdot / \& \mu^{* * *}$ &.$- / 9 r^{* *}$ & $\cdot / r \Lambda^{\mathrm{ns}}$ & وزن هزار دانه \\
\hline 1 & $\cdot / \mu \cdot n s$ & $\cdot \mid \mathrm{N}^{* *}$ & $-\cdot / 0 \cdot^{*}$ &.$-|0|^{*}$ & $-\cdot \Delta r^{*}$ & $-\cdot / 19^{n s}$ & عملكرد دانه \\
\hline
\end{tabular}

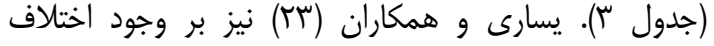

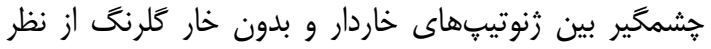

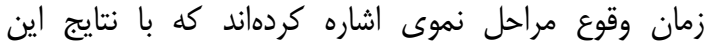

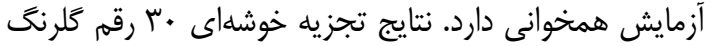

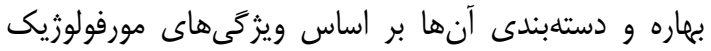

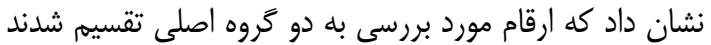

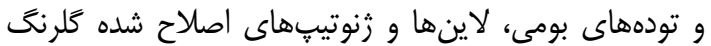

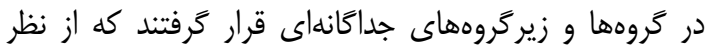
صفات مورفولوزى تنوع بسيارى در بين آنها مشاهده شدأهد (1).

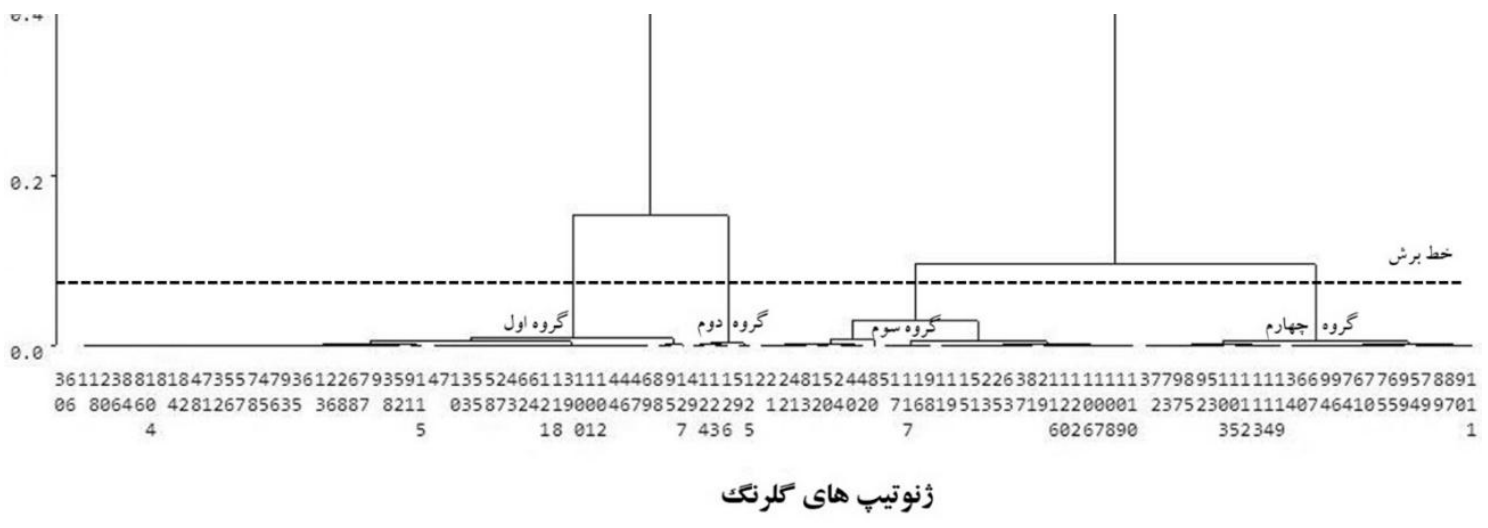

شكل 1- تجزيه خوشهاى (كلاستر) VVI زنوتيڤ گلرنخ بر اساس هفت صفت مورد بررسى (نقطه برش بر اساس يرش ناتمانى فاصله ادغام

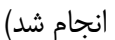

Figure 1. Cluster analysis (cluster) 127 safflower genotypes based on 7 measured traits (cutting point based on the jump of the merger distance) 
جدول عا- نتايج تجزيه خوشهاى (كلاستر) و گروه بندى زنوتيڤهاى گلرنح بر اساس صفات مورد مطالعه Table 4. Results of cluster analysis and classification of safflower genotypes based on studied traits

\begin{tabular}{|c|c|c|c|c|c|c|c|c|}
\hline (كرم در متر & وزن هزار (گرم) & 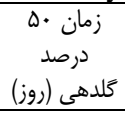 & كلدهى (روز) & خاردار & 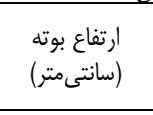 & كلجهنا & شماره (نام) زنوتيڤها & \\
\hline 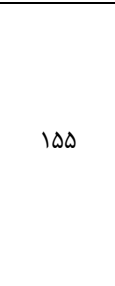 & r/\&q9 & וזוr & Tre & خاردار & ( م • متوسط متر) & رثثراً زردد & 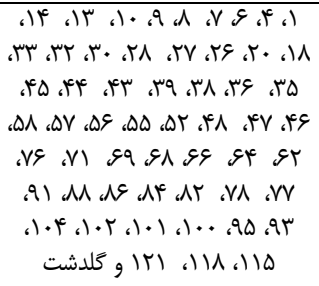 & $\begin{aligned} & \\
& \\
& \end{aligned}$ \\
\hline $1 \wedge 8$ & r/IF & 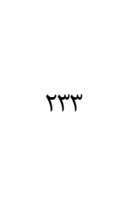 & trA & خدار & 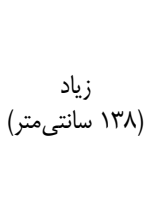 & اكثراً قرمز & 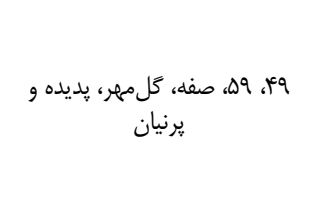 & (و زروه دوم \\
\hline $1 \mathrm{FV}$ & r/VFA & ru. & THA & خاردار & 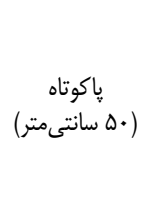 & اكثراً زرد & 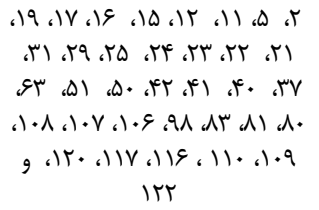 & 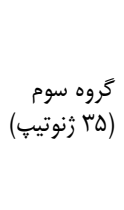 \\
\hline IQT & r/gqr & וודץ & 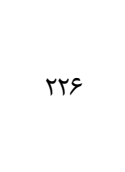 & خاردار & 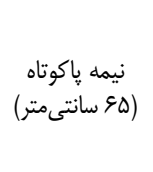 & و زبراً & 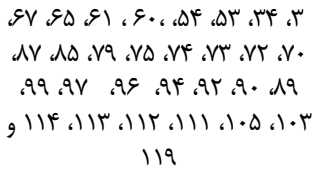 & 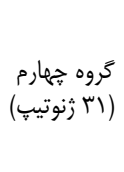 \\
\hline
\end{tabular}

به دليل همبستكى مثبت و بالاى عملكرد دانه با مؤلفه دوم،

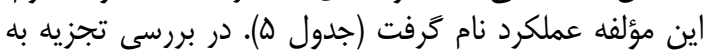

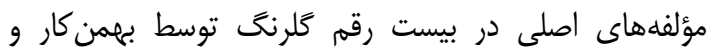

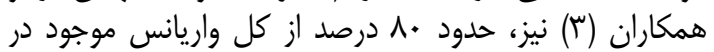

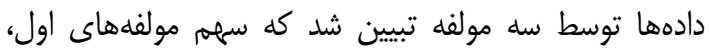

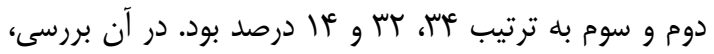

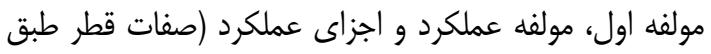

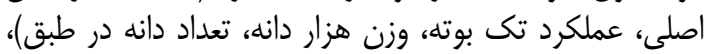

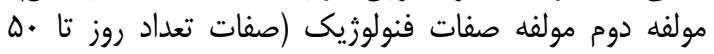

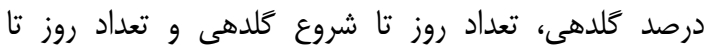

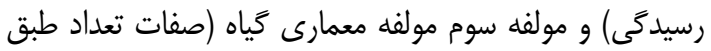

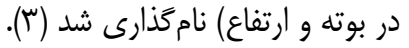

تتايج تجزيه به مؤلفههاى اصلى نشان داد كه سه مؤلفه

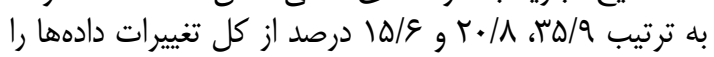

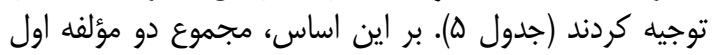

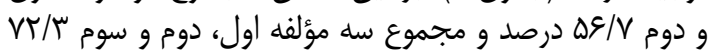

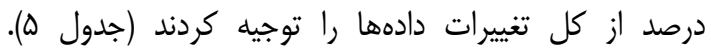

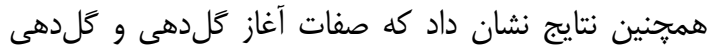

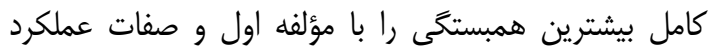

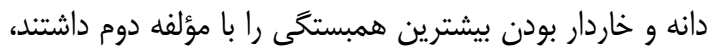

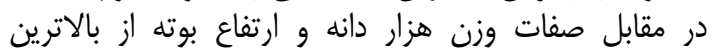

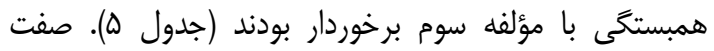

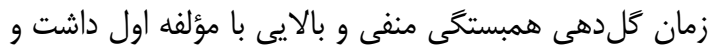

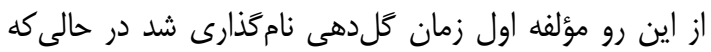

جدول ه- بردارهاى ويثره براى هفت صفت ارزيابى شده در زنوتيِهاى كلرنى با استفاده از تجزيه به مؤلفهاى اصلى Table 5. Eigenvector for seven traits in safflower genotypes using principal component analysis

\begin{tabular}{|c|c|c|c|}
\hline مؤلفه سوم & مؤلفه دوم & مؤلفه اول & صفت \\
\hline$.|| Q \mid$ & ת & $\cdot 1 \cdot V \mathrm{~V}$ & رنگ \\
\hline.$/ \mathrm{AV}$ &.$- / 1 \Lambda \mu$ &.$- / 94 \mathrm{~T}$ & أغاز كلدهي \\
\hline.$/ 1 n$ & $-\cdot / I V \Delta$ & $-\cdot / 941$ & كلدهى كامل \\
\hline - / Keq & $\cdot|\Delta F|$ & $-\cdot / r I r$ & خاردار بودن \\
\hline$-\cdot \mid 4 \wedge 1$ & . & $-\cdot / r V$ & ارتفاع بوته \\
\hline.$/ w n$ &.$- / \cdot r \Delta$ &.$/ M M$ & وزن هزار دانه \\
\hline .1148 & אחק|. & $-\cdot / .+$ & عملكرد دانه \\
\hline $\mid \omega / 9$ & $r \cdot / \Lambda$ & $\Gamma \Delta / 9$ & درصد تغيرات توجيه شده توسط مؤلفهها \\
\hline
\end{tabular}

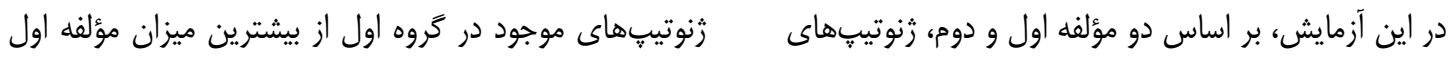

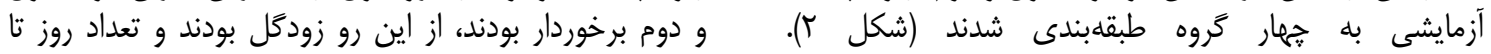




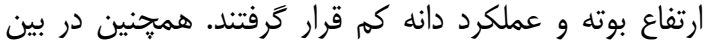

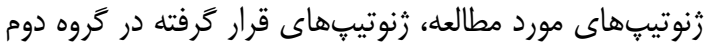

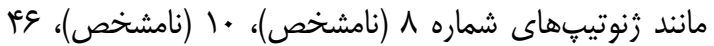

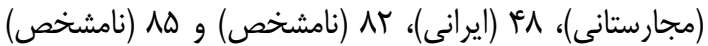

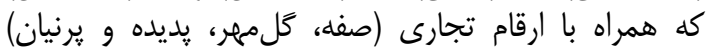
طبقهبندى شده بودند مى توانواندا جها

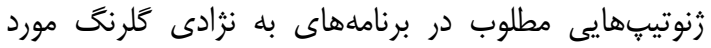

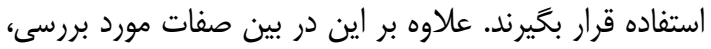

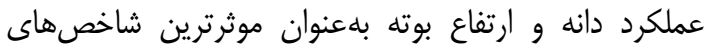

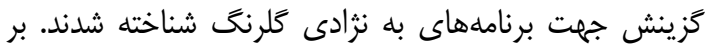

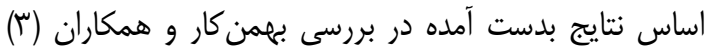

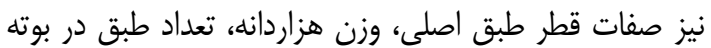

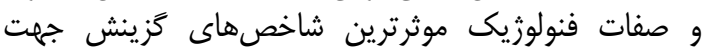

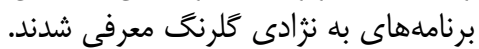

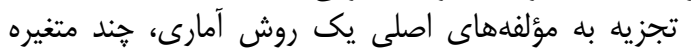

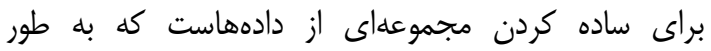

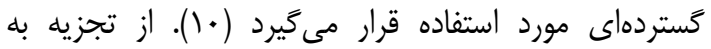

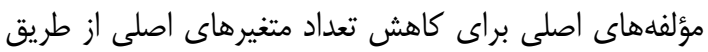

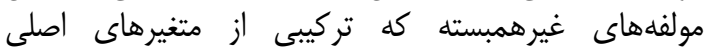

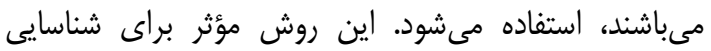

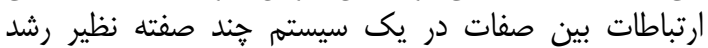

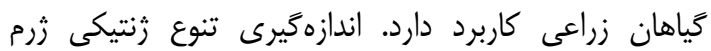

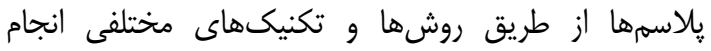

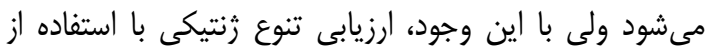

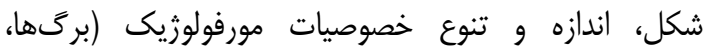

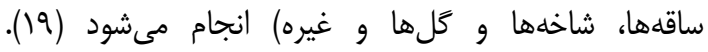

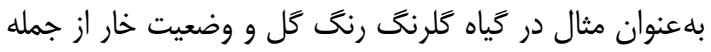

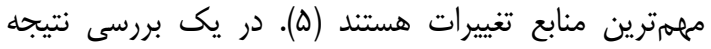

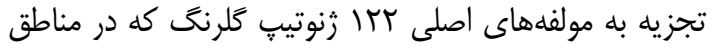

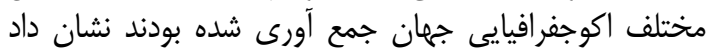

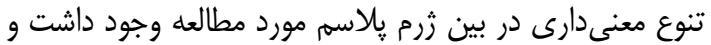

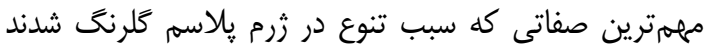

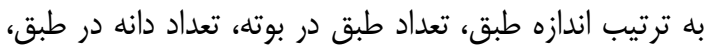

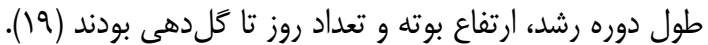

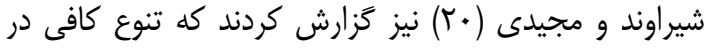

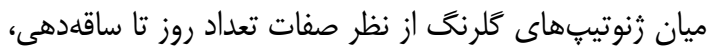

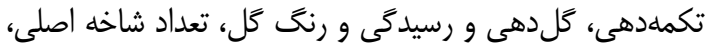

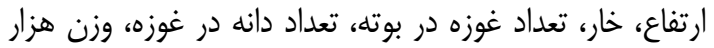

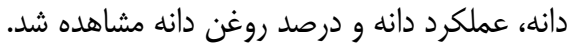

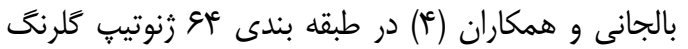

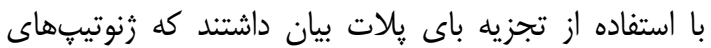

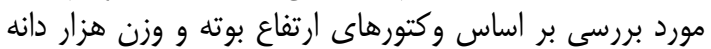

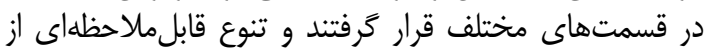
اين نظر داشتند.

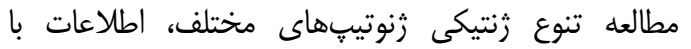

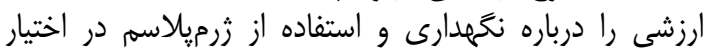

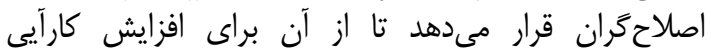
برنامههاى اصلاحى استفاده كنند (ع).

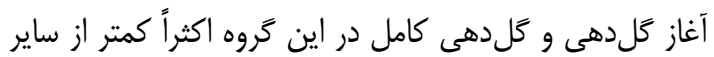

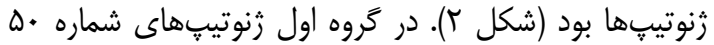

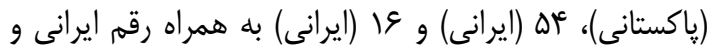

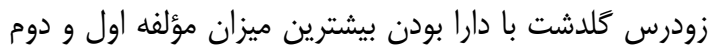

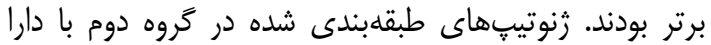

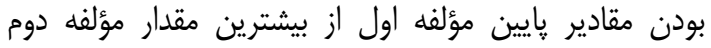

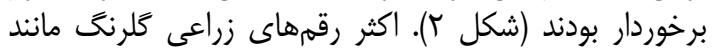

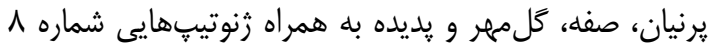

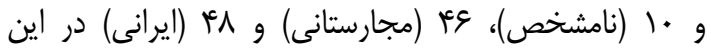

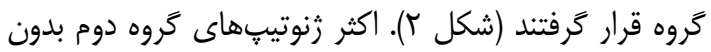

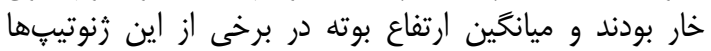

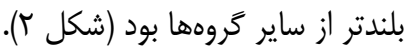

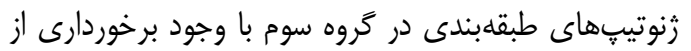

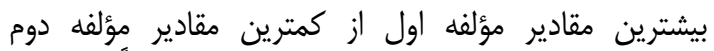

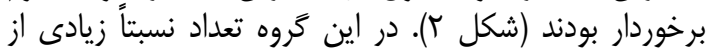

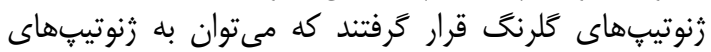

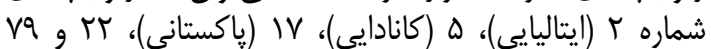

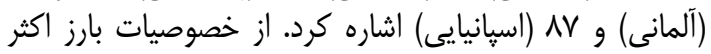

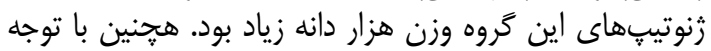

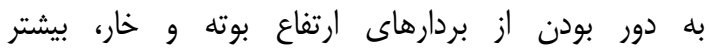
رنوتيِهاى موجود در اين گروه پاكوتاه و خاردار بودند (شكل

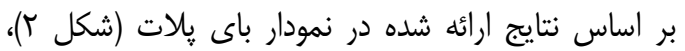

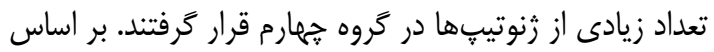

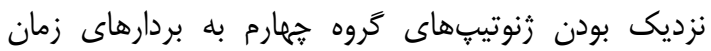

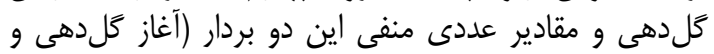
كل كلهى كامل) اكثر زنوتيبها

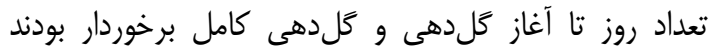

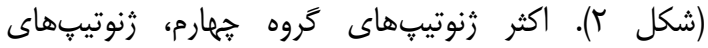

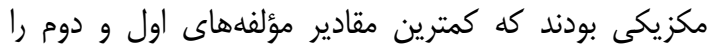

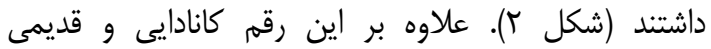
Saffire

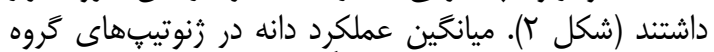

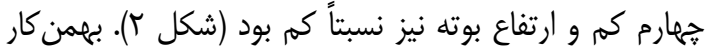

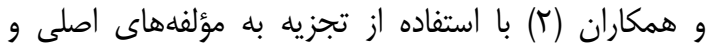

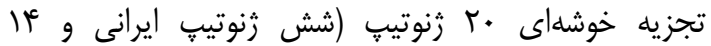

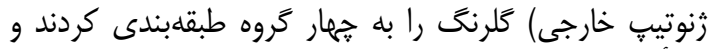

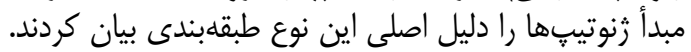

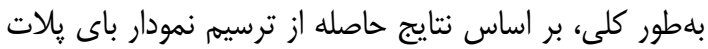

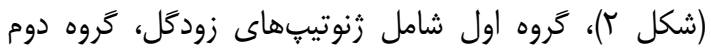

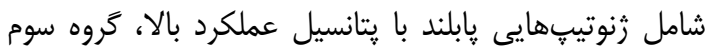

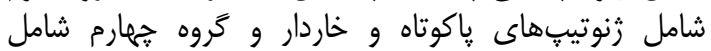

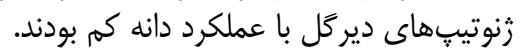

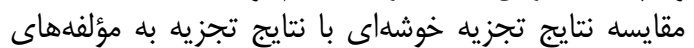

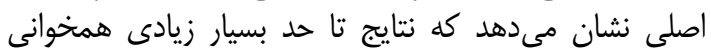

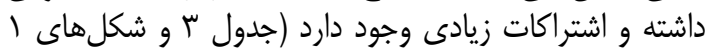

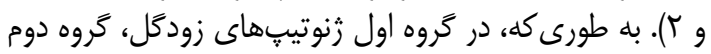

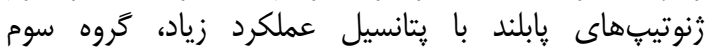

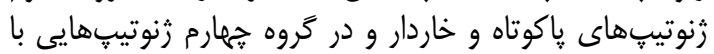




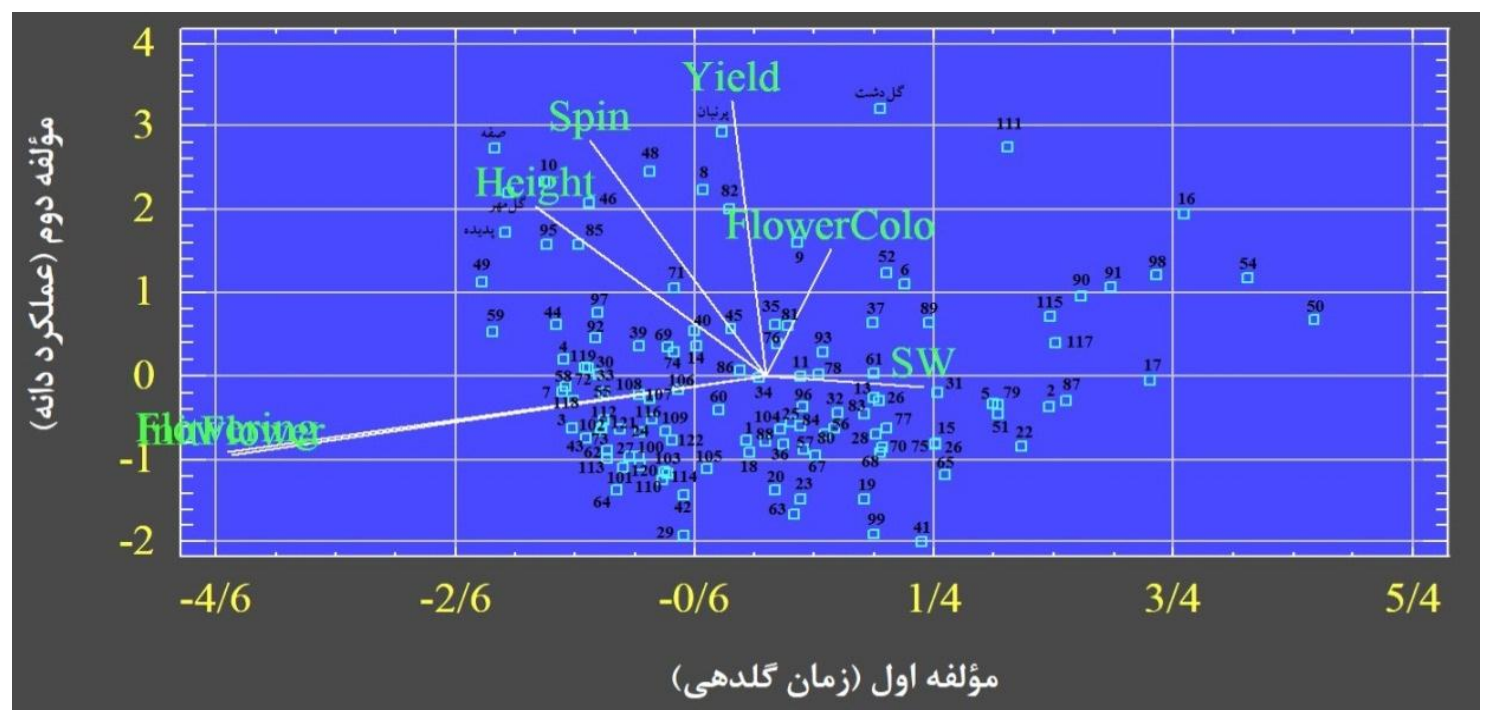

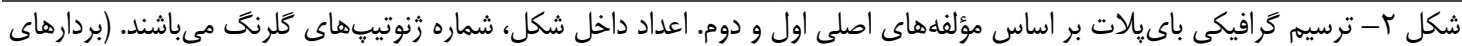

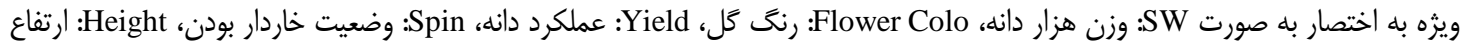

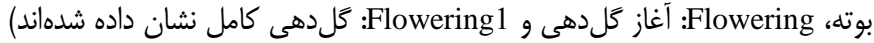

Figure 2. Graphic drawing biplot based on two first components. The numbers inside the figure indicate the numbers of safflower genotyps (Eigenvectors are briefly described as follows sw: 1000 seed weight, Flower Colo: Flower Color, Yield: Seed yield, Spin: spiny or spinless, Height: plant height, Flowering: Number of days to flowering and Flowering1: Number of days until full flowering)

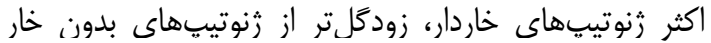

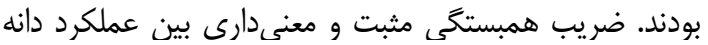

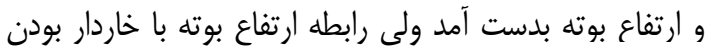

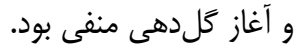

$$
\text { تشكر و قدردانى }
$$

بدينوسيله از زحمات و همكارى وآى جناب آقاى مهندس

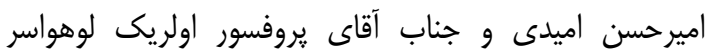
(Ulrike Lohwasser) كياهان زراعى (IPK) كشور آلمان كمال تشكر و قدردانى به

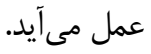

\begin{abstract}
بلهطور كلى تنوع زنتيكى قابلماحظهاى در زرم پالاسم

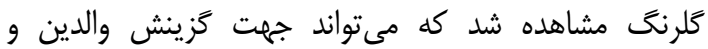

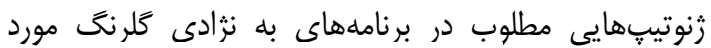

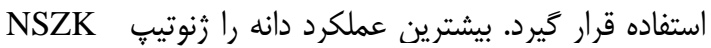

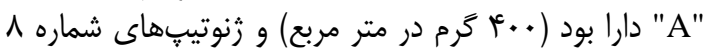

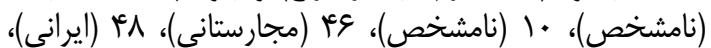

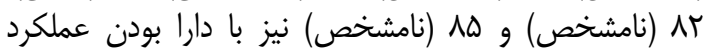

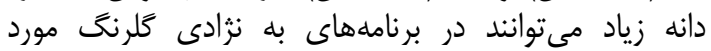

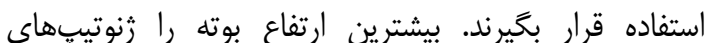

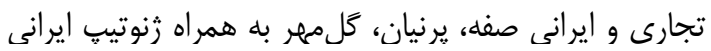

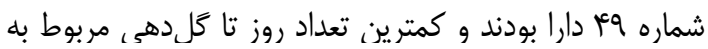

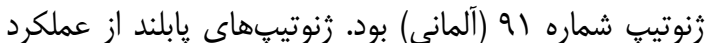
دانه بهترى در مقايسه با زنوتيڤهاى
\end{abstract} cultivars using morphological characters. Advances in Bioresearch, 4: 125-131.

2. Bahmankar, M., D. Ahmadi Nabati and M. Dehdari. 2017. Genetic relationships among Iranian and exotic safflower using microsatellite markers. Journal of Crop Science and Biotechnology, 20(3): 159165.

3. Bahmankar, M., D. Ahmadi Nabati and A. Dehdari. 2013. Study of yield and yield components in safflower using principal component analysis. National conference of passive defense in agriculture. Qeshm Island, Iran's Leading Iranian Science and Technology Co-operative (In Persian).

4. Baljani, R., F. Shekari and N. Sabaghnia. 2015. Biplot analysis of trait relations of some safflower (Carthamus tinctorius L.) genotypes in Iran. Crop Research, 50(1-3): 63-73.

5. Bradley, V.L., R.L. Guenthner, R.C. Johnson and R.M. Hannan. 1999. Evaluation of safflower germplasm for ornamental use. In: Janik, J. (ed.) Perspectives on New Crops and New Uses, 433-435 pp., ASHS Press, Alexandria, USA.

6. Gholami, M., N. Sabaghnia, M. Nouraein, F. Shekari and M. Janmohammadi. 2018. Cluster analysis of some safflower genotypes using a number of agronomic charachteristics. Journal of Crop Breeding, 10(25): 159-166 (In Persian). 
7. Jan, H.U., M.A. Rabbani and Z.K. Shinwari. 2012. Estimation of genetic variability in turmeric (Curcuma longa L.) germplasm using agro-morphological traits. Pakistan Journal of Botany, 44(SI1): 231-238.

8. Jaradat, A.A. and M. Shahid. 2006. Patterns of phenotypic variation in a germplasm collection of Carthamus tinctorius L. from the Middle East. Genetic Resources and Crop Evolution, 53: 225-244.

9. Kaffka, S.R. and T.E. Kearney. 1998. Safflower production in California. University of California, Davis, Division of Agriculture and Natural Resources. Publication 21565, Oakland, USA, 29 pp.

10. Leilah, A.A. and S.A. Al-Khateeb. 2005. Statistical analysis of wheat yield under drought conditions. Journal of Arid Environments, 61: 483-496.

11. Mahmood, T., S. Muhammad and Z.K. Shinwari. 2010. Molecular and morphological characterization of Caralluma species. Pakistan Journal of Botany, 42: 1163-1171.

12. Mirabadi, A., M. Hagh Panah, K. Forozan and S. Talaee. 2018. Evaluating multivariate analysis some of quantitative traits in imported safflower (Carthamus tinctorius L.) genotypes in Sari location. Journal of Crop Breeding, 10(28): 162-170 (In Persian).

13. Morris, J.B. and S.L. Greene. 2001. Defining multiplease germplasm collection for genus Trifollium. Crop Science, 41: 893-901.

14. Omidi, A.H. and F. Javidfar, 2011. Safflower. Seed and Plant Improvement Institute. 117 pp (In Persian).

15. Omidi, A.H., H. Khazaei and S. Hongbo. 2009. Variation for some important agronomic traits in 100 spring Safflower (Carthamus tinctorius L.) genotypes. American-Eurasian Journal of Agricultural \& Environmental Sciences, 5(6): 791-795.

16. Pavithra, K.P., S. Rajesh, Y. Patil Harijan and G.K. Nishanth. 2016. Correlation and path analysis studies in Safflower (Carthamus tinctorius L.) germplasm. Research Journal of Agricultural Sciences, 7(2): 428-432.

17. Poordad, S. 2006. Safflower. Sepehr Publication. 123 pp (In Persian).

18. Sharifnabi, B. and G. Saeidi. 2004. Preliminary evaluation of different genotypes of Safflower (Carthamus tinctorius L.) to Fusarium root rot disease. Journal of Water and Soil Science, 8(3): 219227 (In Persian).

19. Shinwari, Z.K., H. Rehman and M. Ashiq Rabbani. 2014. Morphological traits based genetic diversity in safflower (Carthamus tinctorius L.). Pakistan Journal of Botany, 46(4): 1389-1395.

20. Shiravand, R. and M. Maiidi. 2014. Drought tolerance of wild and cultivated species of safflower and assessment of morphological variation. Iranian Journal of Field Crops Research, 12(4): 738-750 (In Persian).

21. Usha Kiran, B., N. Mukta, P. Kadirvel, K. Alivelu, S. Senthilvel, P. Kishore and K. Varaprasad. 2017. Genetic diversity of safflower (Carthamus tinctorius L.) germplasm as revealed by SSR markers. Plant Genetic Resources, 15(1): 1-11.

22. Weiss, E.A. 2000. Oilseed crops. 2th edn. Blackwell Science Ltd., Oxford, London, Berlin Carlton, Paris. 364 pp.

23. Yasari, T., M. Shahsavari, A. Barzegar and A.H. Omidi. 1995. Study of developmental stages and relationship between of them and seed yield in ten advanced safflower genotypes. Pajouhesh \& Sazandegi, 68(3): 75-83 (In Persian).

24. Yazdi Samadi, B. 1978. Evaluation of drought resistance in Iranian and foreign safflower cultivars. Iranian Journal of Agriculture Science, 2\&3: 6-10 (In Persian). 


\title{
Study of Genetic Variation in Safflower Germplasm for Early Maturity and Grain Yield using Multivariate Statistical Methods
}

\author{
Seyyed Mojtaba Mosavi Ojagh ${ }^{1}$, Hamid Mozafari ${ }^{2}$, Hamid Jabbari ${ }^{3}$ and Behzad Sani ${ }^{4}$ \\ 1 and 4- Ph.D. Student and Assistant Professor, Department of Agronomy, Shahr-e-Qods Branch, Islamic Azad \\ University, Tehran, Iran \\ 2- Assistant Professor, Department of Agronomy, Shahr-e-Qods Branch, Islamic Azad University, Tehran, Iran, \\ (Corresponding author: mozafarihamid@yahoo.com) \\ 3- Assistant Professor of Seed and Plant Improvement Institute (SPII), Agricultural Research, Education and \\ Extension Organization (AREEO), Karaj, Iran \\ $\begin{array}{ll}\text { Received: August 26, } 2018 & \text { Accepted: January 1, } 2019\end{array}$
}

\begin{abstract}
In this study, the genetic diversity of 122 safflower genotypes from the institute of plant genetics and crop plant research (IPK) and International Maize and Wheat Improvement Center (CIMMYT) were evaluated and their agronomic characteristics were compared with five Iranian Safflower cultivars (Sofe, Goldasht, Golmehr, Padide and Parnian). The study was carried out in the Augment design during 2016-2017 at the research field of seed and plant improvement institute in Karaj. The results indicated high genetic variation in the germplasm. Among safflower genotypes, plant height had the most variation. Cluster analysis was divided genotypes into four main clusters. The first cluster included early and spiny genotypes, while the second cluster contained genotypes with high yield potential and high plant height, third cluster consisting of spiny dwarf genotypes and the fourth cluster including semi-dwarf, spiny, relatively early flowering and relatively low grain yield. Based on the principal component analysis, the studied traits were reduced to two components and cumulative variance was $56.7 \%$ for two first components. Accordingly, the first and second components were flowering time and yield, respectively. Based on the results of biplot, genotypes were classified into four groups. Genotypes in the first and second groups had the higher grain yield than others. The highest grain yield was observed in NSZK "A" genotype $\left(400\right.$ g.m $\left.{ }^{-2}\right)$. Iranian commercial cultivar including Sofe, Parnian and Golmehr and Iranian genotype No. 49 showed the maximum plant height. The lowest number of days to flowering was observed in genotype No. 91 (German). Tall genotypes had more grain yield than dwarf. Most spiny genotypes were early flowering than spineless genotypes. Overall, the results showed that there is a considerable genetic variation in safflower germplasm that can be used to select parents and desirable genotypes in safflower breeding programs.
\end{abstract}

Keywords: Biplot Analysis, Cluster Analysis, Early Flowering, Plant Height, Spin 